\title{
O equilíbrio estático e dinâmico em pacientes com Parkinson submetidos a fisioterapia aquática
}

\author{
Static and dynamic balance in patients with Parkinson \\ submitted to aquatic physical therapy
}

\section{Equilibrio estático y dinámico en pacientes con Parkinson presentado a fisioterapia acuática}

\begin{abstract}
Cristiane de Fátima Pimenta da Costa ${ }^{1}$, Náthila Lorrana Silva Cardoso ${ }^{1}$, Michelly Beatriz Fernandes da Silva ${ }^{1}$, Stephanie Araujo Chucre de Lima1 ${ }^{1}$, Izabelle Chrystine Sousa da Silva1, Rosa Costa Figueiredo ${ }^{2}$, Renata Amanajás de Melo², César Augusto de Souza Santos ${ }^{2}$, George Alberto da Silva Dias ${ }^{2}$
\end{abstract}

1.Graduação em Fisioterapia. Departamento de Ciências do Movimento Humano. Universidade do Estado do Pará (UEPA), Belém-PA, Brasil.

2.Fisioterapeuta. Departamento de Ciências do Movimento Humano. Docente da Universidade do Estado do Pará (UEPA), Belém-PA, Brasil.

\section{Resumo}

Objetivo. Avaliar o equilíbrio estático e dinâmico de pacientes com Parkinson submetidos a Fisioterapia aquática. Método. Trata-se de um estudo experimental que utilizou uma amostra composta por 5 indivíduos com DP, com idades entre 40 a 70 anos, apresentando função cognitiva preservada, pressão arterial controlada e ausência de enfermidades como infecções, epilepsia, processos inflamatórios, entre outros. A avaliação foi realizada por meio do Teste de Alcance Funcional (TAF), o Teste Time Up and Go (TUG) e o Step Teste (ST). O procedimento terapêutico foi realizado em 5 fases: Aquecimento, alongamento, fortalecimento, treino de equilíbrio e relaxamento. Resultado. Foram incluídos 5 indivíduos do sexo masculino, com média de idade de $67,40 \pm 8,08$ anos. Antes do tratamento foi observado uma correlação entre o TUG e TAF $(r=-0,9 ; p=0,03)$, entre TUG e ST $(r=0,9 ; p=0,03)$ e não foi notado correlação entre o TAF e ST $(r=-0,7 ; p=0,18)$. Após as sessões, deixou de existir correlação entre o TUG e o TAF $(r=-0,7 ; p=0,18)$, permaneceu a correlação significante para TUG e ST $(r=0,9$; $p=0,03)$ e sem correlação entre TAF e ST $(r=-0,4 ; p=0,50)$. Conclusão. Constatou-se que a Fisioterapia Aquática não apresentou melhora no equilíbrio estático e dinâmico, no entanto contribuiu positivamente na melhora da mobilidade em meio terrestre.

Unitermos. Fisioterapia; Equilíbrio Postural; Doença de Parkinson

\begin{abstract}
Objectives. To evaluate the static and dynamic balance of patients with Parkinson's Disease (PD) who underwent aquatic physical therapy. Method. This is an experimental study that used a sample composed by 5 individuals with PD, with aged 40 to 70 years, with preserved cognitive function, controlled blood pressure and absence of diseases such as infections, epilepsy, inflammatory processes, among others. The assessment was performed using the Functional Reach Test (FR), Timed Up and Go Test (TUGT) and the Step Test (ST). The therapeutic procedure was carried out in 5 phases: Warm-up, stretching, strengthening, balance training and relaxation. Results. Five male individuals were included, with a mean age of $67.40 \pm 8.08$ years. Before treatment, a correlation was observed between TUGT and FR $(r=-0.9 ; p=0.03)$, between TUGT and ST $(r=0.9 ; p=0.03)$ and there was no correlation between FR and ST $(r=-0.7 ; p=0.18)$. After the sessions, there was no longer a correlation between TUGT and FR $(r=-0.7 ; p=0.18)$, the significant correlation remained for TUGT and ST $(r=0.9 ; p=0.03)$ and without correlation between FR and ST $(r=-0.4 ; p=0.50)$. Conclusions.
\end{abstract}


It was found that the aquatic physical therapy does not show improvements in the static and dynamic balance, however it contributed positively to improving mobility in the land environment.

Keywords. Physical Therapy Specialty; Postural Balance; Parkinson Disease

\section{Resumen}

Objetivo. Evaluar el equilibrio estático y dinámico de pacientes con Parkinson que se sometieron a fisioterapia acuática. Método. Este es un estudio experimental que utilizó una muestra compuesta por 5 individuos con EP, con edades entre 40 y 70 años, con función cognitiva preservada, presión arterial controlada y ausencia de enfermedades como infecciones, epilepsia, procesos inflamatorios, entre otros. La evaluación se realizó utilizando la Prueba de alcance funcional (TAF), la Prueba de tiempo transcurrido (TUG) y la Prueba de paso (ST). El procedimiento terapéutico se realizó en 5 fases: calentamiento, estiramiento, fortalecimiento, entrenamiento de equilibrio y relajación. Resultado. Se incluyeron cinco individuos masculinos, con una edad media de 67.40土8.08 años. Antes del tratamiento, se observó una correlación entre TUG y TAF $(r=-0.9 ; p=0.03)$, entre TUG y $S T(r=0.9 ; p=0.03)$ y no hubo correlación entre o TAF y ST $(r=-0.7 ; p=0.18)$. Después de las sesiones, ya no había una correlación entre TUG y TAF $(r=-0.7 ; p=0.18)$, la correlación significativa se mantuvo para TUG y ST $(r=0.9 ; p=0.03)$ y sin correlación entre TAF y $S T(r=-0.4 ; p=0.50)$. Conclusión Se encontró que la Fisioterapia Acuática no mostró mejoría en el equilibrio estático y dinámico, sin embargo, contribuyó positivamente en la mejora de la movilidad en el entorno terrestre.

Palabras clave. Fisioterapia Acuática; Equilibrio Postural; Enfermedad de Parkinson

Trabalho realizado na Universidade do Estado do Pará, Belém-PA, Brasil.

Endereço para correspondência: George AS Dias. Universidade do Estado do Pará (UEPA). Rua do Una 156. CEP 66050-540. Telégrafo, Belém-PA, Brasil. Tel: (91) 31311708. E-mail: george@uepa.br

\section{INTRODUÇÃO}

A doença de Parkinson (DP) afeta o Sistema Nervoso Central (SNC) de forma crônica, progressiva e degenerativa, a qual envolve disfunções nos gânglios da base $e$ consequências nos movimentos voluntários e automáticos ${ }^{1}$. Tais consequências ocorrem principalmente devido à morte dos neurônios produtores de dopamina da substância negra². A DP atinge principalmente pessoas do sexo masculino e a partir da quinta década de vida, sendo em média 200 mil brasileiros $^{3}$. Ainda não existem evidências científicas conclusivas da causa dessa degeneração, sendo então uma doença de caráter idiopático e sabe-se que há múltiplas 
condições influenciadoras, dentre elas a predisposição genética, fatores ambientais, entre outros ${ }^{4}$.

As manifestações motoras ou sinais motores presentes abrangem uma série de características clínicas como o tremor de repouso, rigidez muscular, bradicinesia e instabilidade postural ${ }^{5}$. A interação entre esses sinais compromete a agilidade da marcha do indivíduo ${ }^{6}$, sendo necessário então um bom alinhamento corporal, requisitos biomecânicos, amplitude articular e flexibilidade para que haja uma base de sustentação e ação muscular efetiva7. Com o avanço da patologia, o controle postural, o qual é correlacionado ao equilíbrio estático e dinâmico, torna-se mais comprometido, afetando o equilíbrio e consequentemente sua mobilidade funcional, tornando o indivíduo mais dependente e com maior risco de quedas 4 .

A Fisioterapia voltada para a DP tem como objetivo minimizar os problemas motores, manter e reestabelecer a funcionalidade, auxiliando a independência e proporcionando uma melhor qualidade de vida $(\mathrm{QV})^{8}$. A Fisioterapia Aquática (FA) por sua vez é um recurso que se utiliza dos efeitos físicos, fisiológicos e cinesiológicos advindos da imersão do corpo em piscina aquecida como recurso auxiliar da reabilitação ou prevenção de alterações funcionais. A ação terapêutica da água aquecida acarreta diversos benefícios metabólicos. Além disso, um dos efeitos provocados pela imersão em meio hídrico seria o aumento dos níveis de dopamina no SNC, que se mantêm por algumas horas após a imersão, o que é benéfico para o paciente com DP, visto 
que há uma diminuição dos neurônios dopaminérgicos decorrentes da doença?.

A hidrocinesioterapia envolve o exercício físico dentro da piscina aquecida. Beneficiando-se das propriedades hidrodinâmica e hidrostática que a imersão em água proporciona, podendo atuar como facilitadora, suporte ou resistência nos movimentos, estudos evidenciam que em alguns casos é mais eficaz que os exercícios em solo ${ }^{10}$.

A utilização deste recurso terapêutico no tratamento clínico do paciente com DP traz enormes benefícios aos sintomas motores, além de minimizar os riscos de quedas causados pela alteração de equilíbrio nesses pacientes proporcionando maior autonomia, funcionalidade $e$ qualidade de vida ao paciente. Assim, o objetivo deste estudo foi avaliar o equilíbrio estático e dinâmico de paciente com Parkinson submetidos a Fisioterapia aquática.

\section{MÉTODO}

\section{Amostra}

Participaram do estudo 5 indivíduos com DP, conforme demanda espontânea do serviço de Fisioterapia Neurofuncional da Unidade de Ensino e Assistência em Fisioterapia e Terapia Ocupacional (UEAFTO) da UEPA, sendo desenvolvida no Ambulatório de Hidroterapia Professor Benedito José Ribeiro Duarte. A coleta dos dados ocorreu no período de outubro de 2019 a março de 2020 . A seleção dos 
participantes ocorreu por meio da amostragem não probabilística por conveniência.

Como critérios de inclusão, optou-se por indivíduos com diagnóstico de Doença de Parkinson, ambos os sexos, idade entre 40 a 70 anos, que fossem encaminhados do serviço de Fisioterapia Neurofuncional da UEAFTO/UEPA e aceitassem participar da pesquisa.

Foram excluídos aqueles que não apresentassem função cognitiva suficiente para compreensão e interpretação das perguntas, avaliados por meio do Mini Exame do Estado Mental, bem como pacientes com pressão arterial não controlada, infecção grave no sistema gênito urinário, incontinência intestinal e da bexiga, feridas abertas, epilepsia descontrolada, doença contagiosa através da água e do ar, hipersensibilidade a produtos químicos utilizados na manutenção da água da piscina, tímpanos perfurados, tendência para labirintite e fase aguda de processos inflamatórios, traumáticos ou hemorrágicos.

A pesquisa teve início após aprovação do Comitê de Ética em Pesquisa da Universidade do Estado do Pará (UEPA) (Parecer no 3.959.122). Trata-se de um estudo experimental, seguindo as normas da Resolução 466/12 do Conselho Nacional de Saúde, relativa à pesquisa com seres humanos. Todos os participantes assinaram o Termo de Consentimento Livre e Esclarecido. 


\section{Procedimento}

Inicialmente os participantes submeteram-se a uma avaliação inicial coletando informações sociodemográficas, ocupacionais, relações familiares e conjugais, relato sobre qualidade de vida e atividade de vida diária, morbidades e sinais vitais.

Posteriormente, utilizou-se na pesquisa testes rápidos e de fácil aplicabilidade em ambiente reservado, por meio do Teste de Alcance Funcional Anterior (TAF), o Teste Timed up and go (TUG) e o Step teste.

O TAF avalia a capacidade do indivíduo de se deslocar dentro do limite da estabilidade anterior, muito utilizado para avaliar risco de quedas. Com uma fita métrica fixada à parede, em paralelo ao chão, colocada na direção do acrômio do participante, estando perpendicular à parede, posicionado ao início da fita métrica. Inicia-se com ombro flexionado a $90^{\circ}$, cotovelos estendidos e punhos na posição neutra. 0 indivíduo inclina-se para frente sem tocar o instrumento e, posteriormente, se observa o deslocamento sobre a fita. Como resultado calcula-se a média, após três tentativas. Deslocamentos inferiores a $15 \mathrm{~cm}$ apontam fragilidade e risco de quedas ao paciente em questão ${ }^{11}$.

O TUG avalia o equilíbrio funcional e a mobilidade por meio da quantificação do tempo em segundos de uma sequência de ações ordenadas ao paciente. O participante é instruído a levantar-se de uma cadeira, caminhar por uma distância de 3 metros, virar, voltar em direção à cadeira e sentar-se novamente. A cronometragem inicia-se ao sinal de 
partida e finaliza-se ao retorno do indivíduo à posição inicial. Como resultado, aqueles indivíduos que realizam em até 10 segundos são considerados independentes e sem risco de quedas. Aqueles que se encontram entre 11 a 20 segundos são considerados independentes parcialmente e com baixo risco de quedas. Já aqueles com tempo superior a 20 segundos, possuem déficit significativo da mobilidade física e inclui risco de quedas ${ }^{11}$.

O Step teste avalia requisitos voltados para a marcha e para subida e descida de escadas. Ele envolve transferência de peso de um membro para o outro, sendo então uma avaliação de estabilidade médio-lateral. $O$ teste consiste na ação de colocar, de forma alternada, os pés sobre um degrau de $18 \mathrm{~cm}$ de altura e $40 \mathrm{~cm}$ de profundidade. Obtendo-se o resultado a partir do tempo utilizado para finalizar a tarefa ${ }^{12}$.

O processo de coleta dos dados se deu apenas por um único pesquisador. A avaliação inicial ocorreu em outubro de 2019 e a avaliação final em março de 2020.

Houve um período pequeno de coleta de dados pois ocorreram situações que não estão no domínio dos pesquisadores. Problemas técnicos no aquecedor e obras para melhorar o serviço adiaram o início do estudo e podem ter influenciado em algumas desistências por serem desmarcados alguns dias de atendimento.

Além disso, por causa do isolamento social devido a pandemia do COVID-19, todos os atendimentos foram interrompidos sem previsão de retorno em março de 2020. 
A piscina terapêutica utilizada na pesquisa apresentava dimensões de 8 metros de largura por 8 metros de comprimento e 1,50 metros de profundidade, com água aquecida a $35^{\circ} \mathrm{C}$, com rampa de acesso e barras laterais. As sessões foram realizadas individualmente, com duração de 50 minutos, duas vezes na semana e em dias intercalados.

O procedimento terapêutico foi dividido em 5 fases: 0 aquecimento, o alongamento, o fortalecimento, o treino de equilíbrio e o relaxamento. A primeira fase foi de adaptação ao ambiente aquático utilizando exercícios de caminhadas em diferentes direções perfazendo um total de duas repetições para cada exercício. A segunda fase foi composta por alongamentos ativo-assistidos dos músculos anteriores, posteriores e medias da coxa, bem como posteriores da perna. Todos os alongamentos foram realizados com duração de pelo menos 20 segundos, totalizando 2 repetições para cada músculo ou grupo muscular em cada membro. A terceira fase foi caracterizada por exercícios inicialmente com 2 séries de 10 repetições para cada movimento, sendo eles o de flexão e extensão de quadril e joelho, o de abdução e adução de quadril, bem como o de flexão plantar de tornozelo. Para resistência imposta aos exercícios, utilizou-se flutuadores e resistores. Na quarta fase, foram os exercícios de equilíbrio estáticos e dinâmicos com utilização dos membros superiores e inferiores de forma alternada, rotação de tronco, e subir e descer um step com duração de duas séries de 10 repetições para cada atividade. Por fim, a quinta fase foi o relaxamento aquático com 
duração de pelo menos 5 minutos. Este constou de massagem subaquática por meio de movimentos turbilhonares e a utilização da técnica da dança da respiração do método Watsu, onde o terapeuta sincroniza a sua respiração com a do paciente, ajustando os ciclos de expiração e inspiração.

\section{Análise estatística}

Para análise de dados, adotou-se o software Excel $®$ 2010 para entrada dos dados e confecção das tabelas, bem como o BioEstat 5.0 na análise estatística. As variáveis categóricas foram apresentadas como frequências e as numéricas por meio de medidas de tendência central e dispersão. Avaliou-se a significância dos dados pelo teste $t$ Student e o de correlação de Spearman. Adotou-se o nível de significância de $5 \%$.

\section{RESULTADOS}

Primeiramente, participaram da avaliação inicial 9 indivíduos, no entanto, houve quatro desistências. Assim, concluíram a pesquisa apenas 5 indivíduos do sexo masculino, com média de idade de $67,40 \pm 8,08$ anos. A maioria possuía ensino superior completo $(60,0 \%)$, todos (100\%) eram casados, não possuíam ocupação (100\%) e 3 (60,0\%) negava hipertensão arterial sistêmica (HAS), conforme mostra a Tabela 1. 
Tabela 1. Características dos pacientes com Doença de Parkinson.

\begin{tabular}{lcc}
\hline \multicolumn{1}{c}{ Variáveis } & $\mathbf{n}$ & $\%$ \\
\hline $\begin{array}{l}\text { Escolaridade } \\
\text { Ensino médio }\end{array}$ & 2 & 40,0 \\
$\quad$ Ensino superior completo & 3 & 60,0 \\
$\begin{array}{l}\text { Estado Civil } \\
\quad \text { Solteiro }\end{array}$ & \\
$\quad$ Casado & - & - \\
$\begin{array}{l}\text { Ocupação } \\
\text { Sim }\end{array}$ & 5 & 100,0 \\
$\quad$ Não & - & - \\
Outros problemas & 5 & 100,0 \\
$\quad$ HAS & & \\
$\quad$ Nega HAS & 2 & 40,0 \\
\hline (-) Dados numéricos igual a zero, (HAS) Hipertensão arterial sistêmica.
\end{tabular}

(-) Dados numéricos igual a zero, (HAS) Hipertensão arterial sistêmica.

Com relação as variáveis relacionadas ao equilíbrio, foi possível notar que não houve melhora significativa. No entanto, levando em consideração apenas a média do teste, algumas variáveis como observado para o TUG, os indivíduos obtiveram redução de 3,51 segundos após as sessões de Fisioterapia (Tabela 2). Em uma primeira análise, apesar de parecer pouco, para indivíduos idosos, esta redução é clinicamente bem importante.

Tabela 2. Análise do equilíbrio estático e dinâmico de pacientes com Parkinson.

\begin{tabular}{lccc}
\hline Variáveis & Antes & Depois & P-valor \\
\hline TUG & $17,73 \pm 8,51$ & $14,22 \pm 5,06$ & 0,09 \\
TAF & $23,50 \pm 9,84$ & $20,00 \pm 9,64$ & 0,12 \\
ST & $3,46 \pm 1,24$ & $3,63 \pm 0,74$ & 0,66 \\
\hline (TUG) Timed up and $90:$ (TAF) Teste de Alcance Funcional Anterior: (ST) Step teste
\end{tabular}


Quando se buscou a correlação das variáveis estudadas, foi possível observar resultados interessantes. Antes de iniciar a fisioterapia aquática, os participantes apresentaram correlação significante entre o TUG e TAF ( $r=-$ $0,9 ; p=0,03)$, implicando dizer que, quanto pior é o equilíbrio dinâmico, pior é o equilíbrio estático, ou seja, uma variável influencia na outra. Foi observado também correlação significante entre TUG e ST $(r=0,9 ; p=0,03)$, ou seja, mais uma vez o equilíbrio dinâmico interferindo no equilíbrio estático. Porém, não foi notado correlação entre o TAF e ST $(r=-0,7 ; p=0,18)$.

Ao se realizar estas mesmas correlações após as sessões de Fisioterapia aquática, observou-se resultados intrigantes. Deixou de existir correlação entre o TUG e o TAF $(r=-0,7 ; p=0,18)$, implicando dizer que agora uma variável não influencia na outra, conforme mostra a Tabela 3. Podendo assim levantar a hipótese de que houve melhora nesta variável por meio do tratamento proposto, o que não foi observado na primeira análise realizada apenas com comparações. Porém, permaneceu a correlação significante para TUG e ST $(r=0,9 ; p=0,03)$ e sem correlação entre TAF e ST $(r=-0,4 ; p=0,50)$.

Tabela 3. Correlação entre o equilíbrio dinâmico e estático de pacientes com Parkinson.

\begin{tabular}{lcccc}
\hline \multirow{2}{*}{ Variáveis } & \multicolumn{2}{c}{ Antes } & \multicolumn{2}{c}{ Depois } \\
\cline { 2 - 5 } & $\mathbf{r}$ & $\mathbf{p}$-valor & $\mathbf{r}$ & $\mathbf{p}$-valor \\
\hline TUG $\times$ TAF & $-0,9$ & 0,03 & $-0,7$ & 0,18 \\
TUG $\times$ ST & 0,9 & 0,03 & 0,9 & $\mathbf{0 , 0 3}$ \\
TAF $\times$ ST & $-0,7$ & 0,18 & $-0,4$ & 0,50 \\
\hline
\end{tabular}

(TUG) Timed up and go; (TAF) Teste de Alcance Funcional Anterior; (ST) Step teste. 


\section{DISCUSSÃO}

A DP, em diversas pesquisas citadas neste artigo, é demonstrada como um fator que interfere nas tarefas funcionais do indivíduo devido a mesma afetar a mobilidade, equilíbrio e postura ${ }^{9}$. Esta doença diminui a agilidade dos pacientes em atividades que demandam Dupla Tarefa (DT) e isto constata o quanto ela interfere no cotidiano e necessita de intervenções eficazes ${ }^{13}$.

A Fisioterapia aquática é um recurso muito interessante para a melhora de várias características motoras e não motoras de pacientes com DP, conforme é observado em alguns estudos ${ }^{9}$.

Pode-se afirmar que o treino de marcha na água tem efeitos positivos relacionados a velocidade e que, para um resultado clínico positivo na marcha, devem ser associados exercícios para mobilidade e equilíbrio ${ }^{14}$, o que pode ser observado neste estudo em que o protocolo proposto foi pensado não somente para o treino de marcha, mas sim atividades que pudessem treinar o equilíbrio destes pacientes.

Em relação ao protocolo aplicado, os participantes relataram satisfação durante o processo, avaliando-o como dinâmico. Houve relato de que alguns exercícios utilizados no mesmo eram eficazes como estratégia para marcha no solo. Além disso, apesar da fase de relaxamento ter duração média de 5 min, ela conseguia alcançar níveis bons de bemestar, levando muitas vezes os indivíduos a adormecerem. 
Neste estudo, houve um predomínio da participação de indivíduos do sexo masculino, podendo ser um reflexo da suposição de prevalência de acometimento da DP ${ }^{15}$, tendo em vista que não foi utilizado nenhum critério de gênero elegível. Ademais, a média de idade dos participantes estava na faixa etária elencada como fator de risco para a doença ${ }^{1}$.

Sabe-se que a DP se apresenta de forma neurodegenerativa e progressiva, a qual ainda não possui cura. Devido a isso, os pacientes tendem a apresentar um nível de dependência cada vez maior, tornando-os impossibilitados de exercerem suas atividades. Assim, neste estudo todos os participantes não possuíam ocupação, e, nesse contexto, o apoio familiar torna-se essencial ${ }^{16}$.

Além dos fatores já mencionados, a hipertensão arterial sistêmica (HAS) é um dos comprometimentos mais encontrados em pacientes com DP ${ }^{17}$. Isso corrobora com o achado nesta pesquisa, no qual foi encontrado pacientes que declararam possuir HAS.

A DP proporciona alteração da estabilidade postural e déficits no equilíbrio, logo o fisioterapeuta deve buscar alternativas que visem impedir ou retardar essas perdas, sendo uma delas a fisioterapia aquática ${ }^{18}$. Apesar de já ser uma opção bastante utilizada com indivíduos idosos e também para ganho de equilíbrio, os resultados apontam que não houve melhora estatística nas variáveis relacionadas ao equilíbrio. Porém, buscando repercussões clínicas diante dos resultados obtidos, percebe-se que, comparando as médias dos testes aplicados, principalmente no TUG, houve 
redução no tempo do teste. Concluindo-se que melhorou clinicamente o equilíbrio dinâmico destes indivíduos ${ }^{19}$.

Cabe ressaltar que há limitações neste estudo no que se refere a frequência dos participantes. É sabido que houve bastante faltas por variados motivos como enfermidades, falta de acompanhante para trazer aos atendimentos, desistências devido a própria dificuldade de locomoção e outras situações do seu contexto social. Todos estes fatores levantados levaram a um número amostral reduzido. Alguns resultados de outros participantes com DP não entraram neste estudo uma vez que o número de sessões também foi reduzido, o que poderia levar a um viés de resultado.

\section{CONCLUSÃO}

Pode-se constatar que a FA não apresentou melhora significante no equilíbrio estático e dinâmico. Porém, podese perceber que houve resultados clinicamente positivos na melhora da mobilidade no meio terrestre, fato relatado pelos próprios participantes do estudo. Ressalta-se a importância da realização de estudos posteriores, com maior número de sessões e sem interrupções, a fim de reafirmar a eficácia da intervenção da FA no paciente com DP. 


\section{AGRADECIMENTOS}

Agradecemos ao CNPQ (Conselho Nacional de Desenvolvimento Científico e Tecnológico), pelo auxílio financeiro que possibilitou a realização desse estudo.

\section{REFERÊNCIAS}

1.Filippin NT, Martins JS, Libera LBD, Halberstadt BF, Severo AR. Qualidade de vida de sujeitos com doença de Parkinson e seus cuidadores. Fisioter Mov 2014;27:57-66. http://dx.doi.org/10.1590/0103-5150.027.001.AO06

2.Souza CFM, Almeida HCP, Sousa JB, Costa PH, Silveira YSS, Bezerra JCL. A doença de Parkinson e o processo de envelhecimento motor: uma revisão de literatura. Rev Neurocienc 2011;19:718-23. https://doi.org/10.34024/rnc.2011.v19.8330

3.Fernandes I, Andrade Filho AS. Estudo clínico-epidemiológico de pacientes com Doença de Parkinson em Salvador-Bahia. Rev Bras Neurol Psiq 2018;22:45-59.

https://rbnp.emnuvens.com.br/rbnp/article/view/244/141

4.Severiano MIR, Zeigelboim BS, Teive HAG, Santos GJB, Fonseca VR. Effect of virtual reality in Parkinson's disease: a prospective observational study. Arq Neuropsiquiatr 2018;76:78-84. https://doi.org/10.1590/0004-282x20170195

5.Spitz M, Tinoco V, Menezes F, Pereira JS. Análise dos sintomas motores na doença de Parkinson em pacientes de hospital terciário do Rio de Janeiro. Rev Bras Neurol 2017;53:14-8.

http://docs.bvsalud.org/biblioref/2017/12/876870/rbn-533-2-analisedos-sintomas-motores-na-doenca.pdf

6.Lana RC, Álvares LMRS, Nasciutti-Prudente C, Goulart FRP, TeixeiraSalmela LF, Cardoso FE. Percepção da qualidade de vida de indivíduos com Doença de Parkinson através do PDQ-39. Rev Bras Fisioter 2007; 11:397-402.

https://doi.org/10.1590/S1413-

35552007000500011

7. Moreira AC, Silva GAN. Avaliação do equilíbrio e flexibilidade de indivíduos em estágios iniciais de progressão da Doença de Parkinson (dissertação). Uberlândia: Universidade Federal de Uberlândia; 2019, 29p. https://repositorio.ufu.br/handle/123456789/28973

8. Vara AC, Medeiros R, Striebel VLW. O tratamento fisioterapêutico na doença de Parkinson. Rev Neurocienc 2012;20:266-72. https://doi.org/10.34024/rnc.2012.v20.8281

9.Silva DM, Nunes MCO, Oliveira PJAL, Coriolano MGWS, Berenguer FA, Lins OG, et al. Efeitos da fisioterapia aquática na qualidade de vida de sujeitos com a doença de Parkinson. Fisioter Pesq 2013;20:17-23. https://doi.org/10.1590/S1809-29502013000100004 
10.D'Agostini CM, Canabarro A, Jorge MSG, Wibelinger LM. Intervenção hidrocinesioterapêutica no equilíbrio postural e na qualidade de vida de mulheres com Fibromioalgia. Rev CPAQV 2018;10:1-12. https://doi.org/10.36692/cpaqv-v10n2-4

11.Karuka AH, Silva JAMG, Navega MT. Análise da concordância entre instrumentos de avaliação do equilíbri corporal em idosos. Rev Bras Fisioter 2011;15:460-6. https://doi.org/10.1590/S141335552011000600006

12.Castro PMMA, Magalhães AM, Cruz ALC, Reis NSRD. Testes de equilíbrio e mobilidade funcional na predição e prevenção de riscos de quedas em idosos. Rev Bras Geriatr Gerontol 2015;18:129-40. http://dx.doi.org/10.1590/1809-9823.2015.13208

13.Terra, MB, Rosa PC, Torrecilha LA, Costa BT, Ferraz HB, Santos SMS. Impacto da doença de Parkinson na performance do equilíbrio em diferentes demandas atencionais. Fisioter Pesq 2016;23:410-5. http://dx.doi.org/10.1590/1809-2950/16659423042016

14. Costa PS, Bôas ECCV, Fonseca EP. Efetividade do treino de marcha na água para pacientes com doença de parkinson: revisão sistemática. Rev Pesq Fisio 2018;8:551-7. https://doi.org/10.17267/22382704rpf.v8i4.2034

15. Hirsch $L$, Jette $N$, Frolkis $A$, Steeves $T$, Pringsheim $T$. The Incidence of Parkinson's Disease: A Systematic Review and Meta-Analysis. Neuroepidemiology 2016;46:292-300. 1 https://doi.org/0.1159/000445751

16.Faria LJF, Lima PMR, Silva NLP. Resiliência familiar diante do diagnóstico da doença de Parkinson na velhice. Pesq Prát Psicossoc 2019;14:1-18.

http://www.seer.ufsj.edu.br/index.php/revista ppp/article/view/1875 17.Fernandes I, Andrade Filho AS. Estudo clínico-epidemiológico de pacientes com Doença de Parkinson em Salvador-Bahia. Rev Bras Neurol Psiq 2018;22:45-59.

https://rbnp.emnuvens.com.br/rbnp/article/view/244/141

18. Moreira WEM, Cassimiro MS, Rodrigues AP. Fisioterapia aquática como coadjuvante no tratamento de idosos com doença de Parkinson. Rev Edu Saúde 2018;1:96-105.

http://186.194.210.79:8090/revistas/index.php/educacaoesaude/arti cle/view/219

19.Franciulli PM, Souza GB, Albiach JF, Santos KCP, Barros LO, Santos NT, et al. Efetividade da hidroterapia e da cinesioterapia na reabilitação de idosos com histórico de quedas. Est Interdisc Envel 2015;20:67186. https://pesquisa.bvsalud.org/portal/resource/pt/biblio-868923 